\title{
LABVIEW BASED CONVEYOR CONTROL SYSTEM
}

\author{
S. Arvind ${ }^{1}$, Mohit Shah ${ }^{2}$ \\ ${ }^{1}$ Student, Electronics and Instrumentation Department, VIT University, TN, India \\ ${ }^{2}$ Student, Electronics and Instrumentation Department, VIT University, TN, India
}

\begin{abstract}
A conveyor control system project was implemented using software LabVIEW and interfacing electronics such that it mimics the operation of a conveyor used in a bottling plant with beginning, filling and ending stations. Conveyors are used for automated transportation of materials. For this project, the conveyor simulator is equipped with a dc motor mounted directly to an axle for driving the conveyor belt, a freely rotating axle for the opposite side of the conveyor belt. Three IR proximity sensors, positioned at the middle and both the ends, are used to detect the position of bottle on the conveyor. A load sensor is employed at the middle to measure the volume of fluid in the bottle. Suitable displays were incorporated in the software. A PC based control system enables fast communication between controls programming and equipment.
\end{abstract}

Key Words: conveyor control system, LabVIEW, sensors

\section{INTRODUCTION}

Today, graphical user interface based control is gaining more prominence in comparison to command line interface as graphical user interface based control allows for greater efficiency and productivity while interacting with the electronic devices. LabVIEW provides an intuitive development environment to create a custom graphical user interface for the user based on the application. This paper presents a LabVIEW based conveyor control system that can be employed in a bottling plant. Conveyors are especially useful in applications involving the transportation of heavy or bulky materials. The control and monitoring of large overland conveyors in a bottling plant involves a wide variety of electrical disciplines, which need to be carefully integrated and interfaced in order to achieve a successful, reliable and efficient end result. This conveyor consists of three stages. In the first stage, the bottle is detected (conveyor paused) and then moved to the middle stage. In the middle stage, the bottle is filled with fluid using a valve that is electrically controlled by the Arduino, programmed using LabVIEW through the LINX interface. Finally, the bottle is moved to the finishing or the final stage where the bottle exits the conveyor and control system should be aware of the exit. The algorithm is decided in such a way that the control system is able to support multiple bottles on the conveyor. The advantage of a conveyor control system in comparison to a conventional control system in object transfer is its speed and automation.

\section{HARDWARE ARCHITECTURE}

\subsection{Sensors}

IR sensors are used for detection of object/bottle at the specific stage. The signal from the IR sensor serves as an input to the microcontroller (Arduino). The signal is further processed to control the motor and solenoid valve based on the algorithm being used. Load sensor is used to measure the volume of fluid in the bottle.

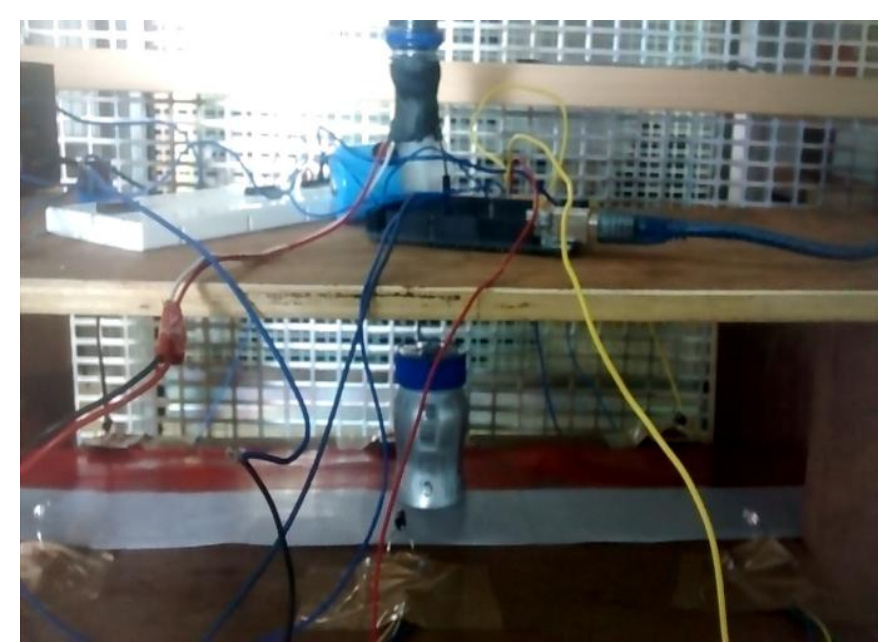

Fig -1: Hardware setup

\subsection{Actuators}

A DC motor connected to the axle is used to drive a conveyor via a roller. A high torque motor is used as it holds control of the whole conveyor. But at the same time, motor torque should not be very high as it will lead to jerks. A solenoid valve is used to control the flow of the fluid. It is switched on when the bottle reaches the filling stage. Appropriate motor and valve driving circuits are used to control the conveyor belt movement.

\subsection{Arduino Mega Board}

The Arduino Mega [1] is a microcontroller board based on the ATmega2560. It has 54 digital input/output pins, 16 analog inputs, a $16 \mathrm{MHz}$ ceramic resonator, a USB connection, a power jack, an ICSP header, and a reset button. It contains everything needed to support the microcontroller. 


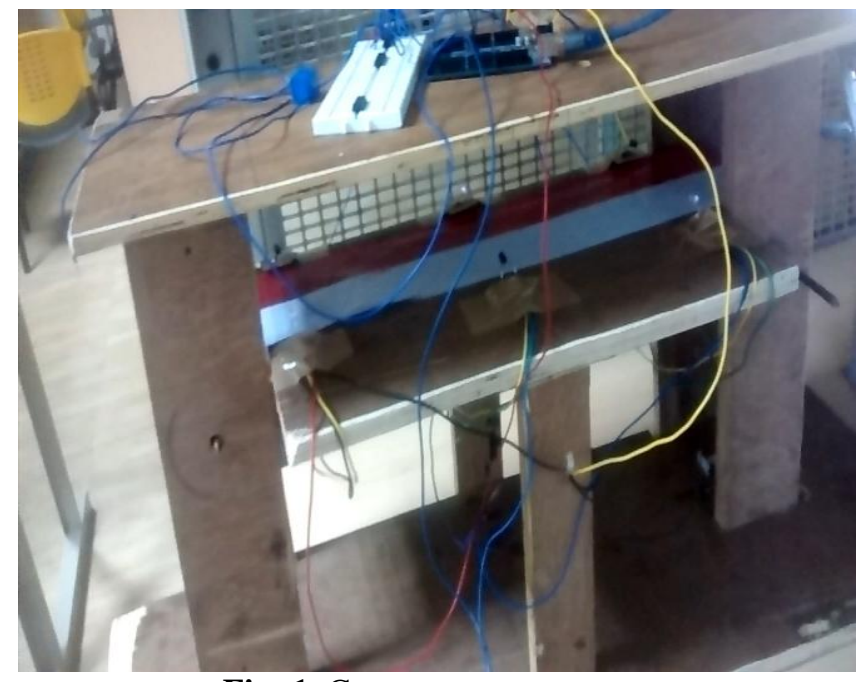

Fig -1: Conveyor system setup

\section{SOFWARE ARCHITECTURE}

\subsection{LabVIEW}

LabVIEW [2] is a system-design platform and development environment for a visual programming language from National Instruments. It basically has two components: front panel and block diagram. Front panel is built using inputs and outputs. The block diagram also known as the back panel contains the graphical source code.

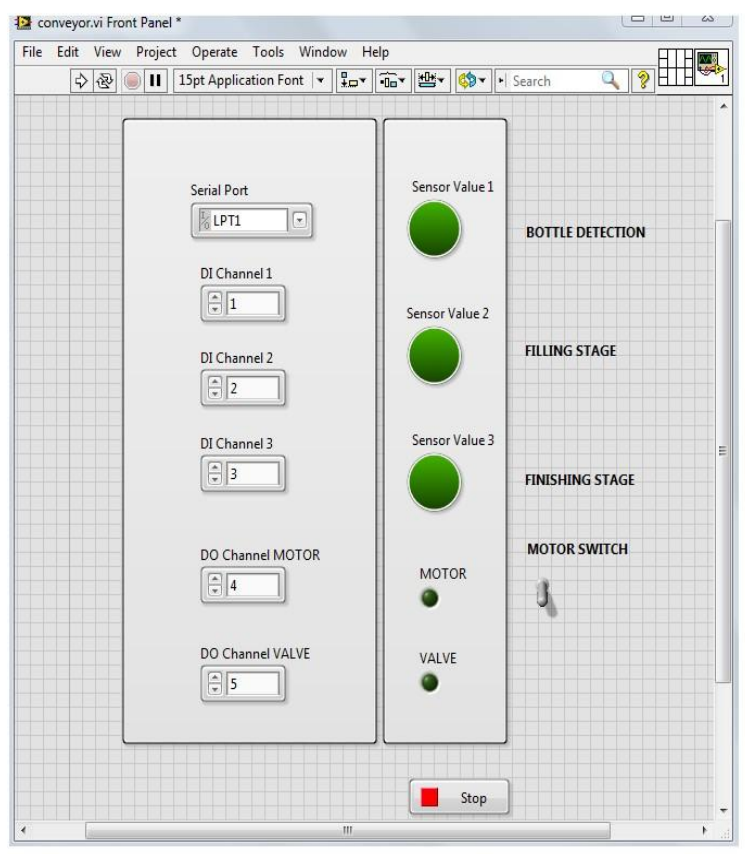

Fig -2: LabVIEW Front Panel

The LINX [3] by LabVIEW MakerHub makes it easy to interface with common embedded platforms such as chipKIT, Arduino, and NI myRIO as well as common sensors including accelerometers, temperature sensors, and ultrasonic distance sensors. Using this toolkit and NI LabVIEW software, control and data acquisition from common embedded platforms is easy and efficient.

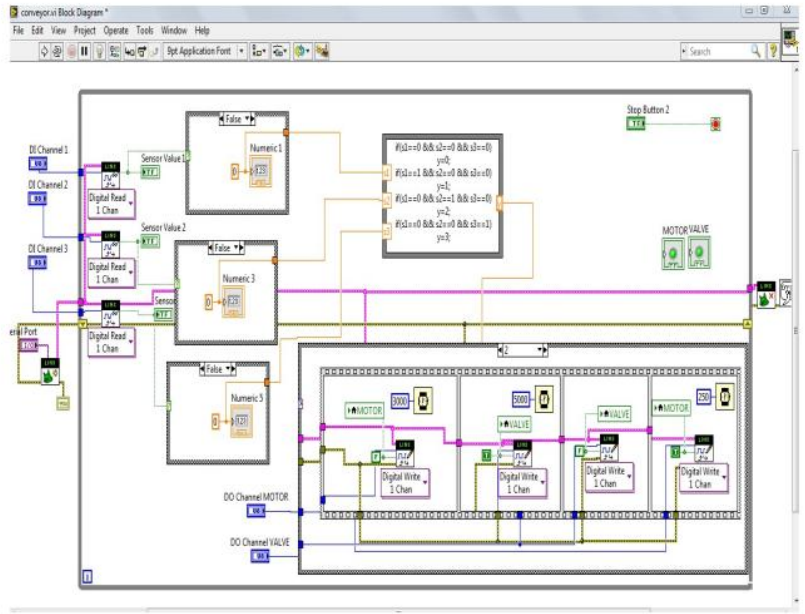

Fig -3: LabVIEW Block Diagram

\section{ALGORITHM}

- Initially, the conveyor system is off and waiting for the motor switch on the user interface to be pressed. As it is pressed, the conveyor starts running and waits for the bottle.

- As the bottle is detected, the conveyor belt pauses for 2 seconds during which detection stage indicator on the user interface is on.

- The bottle is passed to the filling stage by the conveyor, where the solenoid valve is activated until the bottle is filled with the fluid. During this time, filling stage indicator is on. As the bottle is filled to the limit, the load sensor reacts by transmitting a signal to the microcontroller and thus switching the solenoid valve off.

- Finally, the filled bottle is passed to the finishing stage (pausing for 2 seconds) indicating that bottle is about to leave the conveyor system. During this time, finishing stage indicator is on.

- This algorithm works in the case of multiple bottles also such that two or three bottles being on conveyor system at same time.

\section{CONCLUSIONS}

In this paper, LabVIEW based conveyor control system that finds its use in a bottling plant, has been presented. It comprises of three stages namely detection, filling and finishing stage. Through the LINX interface, LabVIEW can be used to develop real time graphical applications. Graphical programming is easy to comprehend, common programming tasks, like debugging, become more intuitive and it also provides the advantages of automatic parallelism and performance. This project can be extended by employing additional features in the user interface

\section{ACKNOWLEDGEMENT}

We would like to thank our coursework faculties for their guidance and provision of the testing facilities. We would also like to thank our friends of VIT University who helped us in completing this project. 


\section{REFERENCES}

[1]. https://www.arduino.cc/en/Main/ArduinoBoardMega2 560

[2]. http://www.ni.com/labview/

[3]. https://www.ni.com/visa/

[4]. https://www.labviewmakerhub.com/doku.php?id=libra ries:linx:start

[5]. David R. Loker, RobertWeissbach, Adam Henry "Conveyor Control System Project" published in "American Society for Engineering Education, 2011"

[6]. "Control System Design Guide" by George Ellis.

[7]. R.M.Nachammai, P.Bhargghavi "Automatic bottle filling system using LabVIEW" published in Proceedings of 4th IRF International Conference on 19th April 2015, Cochin, India 